\title{
Sharing the Load: Stress Redistribution Governs Fracture of Polymer Double Networks
}

\author{
Justin Tauber, Lorenzo Rovigatti, Simone Dussi, and Jasper van der Gucht*
}

Cite This: Macromolecules 2021, 54, 8563-8574

Read Online
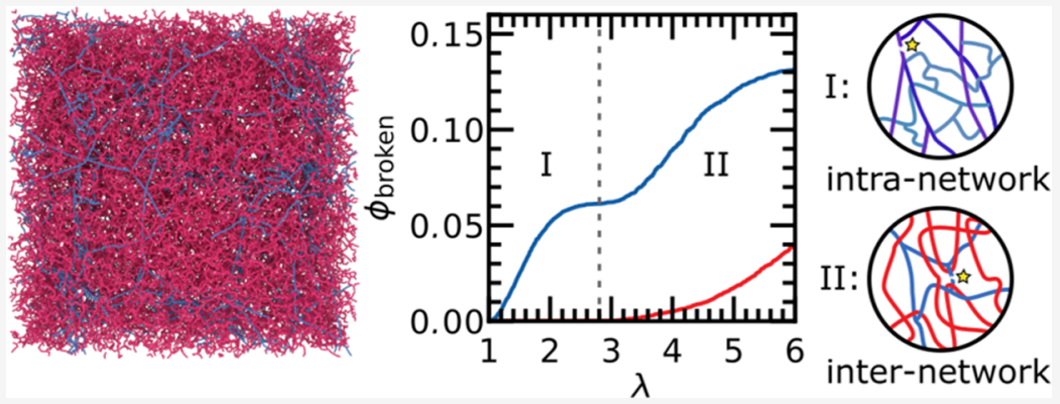

ABSTRACT: The stress response of polymer double networks depends not only on the properties of the constituent networks but also on the interactions arising between them. Here, we demonstrate, via coarse-grained simulations, that both their global stress response and their microscopic fracture mechanics are governed by load sharing through these internetwork interactions. By comparing our results with affine predictions, where stress redistribution is by definition homogeneous, we show that stress redistribution is highly inhomogeneous. In particular, the affine prediction overestimates the fraction of broken chains by almost an order of magnitude. Furthermore, homogeneous stress distribution predicts a single fracture process, while in our simulations, fracture of sacrificial chains takes place in two steps governed by load sharing within a network and between networks, respectively. Our results thus provide a detailed microscopic picture of how inhomogeneous stress redistribution after rupture of chains governs the fracture of polymer double networks.

\section{INTRODUCTION}

By consecutively cross-linking two interpenetrating polymer networks, a composite material is created, which is commonly referred to as a (polymer) double network (DN). ${ }^{1,2}$ In many DNs, the two underlying networks do not share any internetwork cross-linkers and are only topologically constrained at the chain level. ${ }^{1,3}$ For this reason, DNs can be considered as a molecular composite. ${ }^{4} \mathrm{DNs}$ have attracted considerable interest due to the significant enhancement in their (linear) stiffness, strength, and fracture toughness compared to single networks (SNs). ${ }^{1,4-8}$ For example, through this procedure, hydrogels can be constructed that have a mechanical response similar to that of an elastomer. ${ }^{9}$

Experiments reveal that stiff, strong, and tough DNs are created when the first network, or sacrificial network, is stiff and weak, while the second network, or matrix network, is soft and stretchable. ${ }^{9}$ To make networks with these properties, one can vary the type and concentration of monomers and crosslinkers in both networks. ${ }^{8}$ Additionally, these properties can be controlled by swelling the sacrificial network either by introducing a molecular stent ${ }^{3,10}$ or by using the monomer of the second network. ${ }^{2}$ Experiments on a range of systems, varying from elastomers ${ }^{2,4}$ to macroscopic networks, ${ }^{11}$ suggest that the mechanism through which the enhancement occurs is surprisingly general: sharing of load between the two networks via their topological constraints. ${ }^{12}$

The corresponding microscopic picture is that, due to the presence of the matrix chains, the expansion of a (microscopic) crack in a DN requires considerably more energy than in an $\mathrm{SN}^{2,5-7,13}$ As a consequence, fracture of sacrificial chains in $\mathrm{DNs}$ is less likely to lead to the formation of macroscopic cracks and thus global failure. ${ }^{14}$ Instead, the load is transferred (partially) from the sacrificial network to the matrix network surrounding the broken sacrificial polymer chain. ${ }^{4}$ Thus, in a $\mathrm{DN}$, more sacrificial chains can break prior to global failure compared to an SN. As the intact sacrificial chains in these DNs can still resist deformation, the work required for global failure of the material is increased significantly compared to the individual networks. This concept has been termed the sacrificial bond principle ${ }^{12}$ and is widely accepted as the

Received: June 14, 2021

Published: September 16, 2021 
(a)

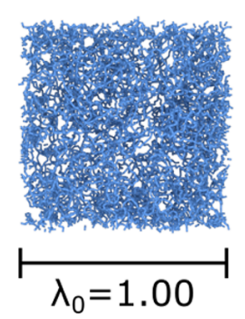

(c)

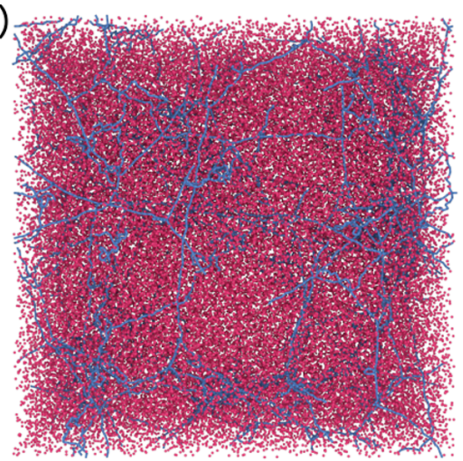

(b)

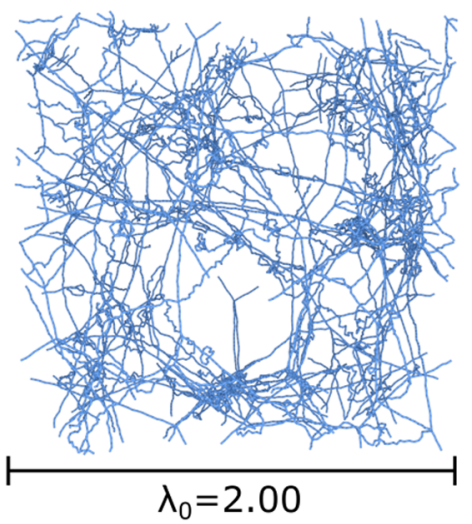

(d)

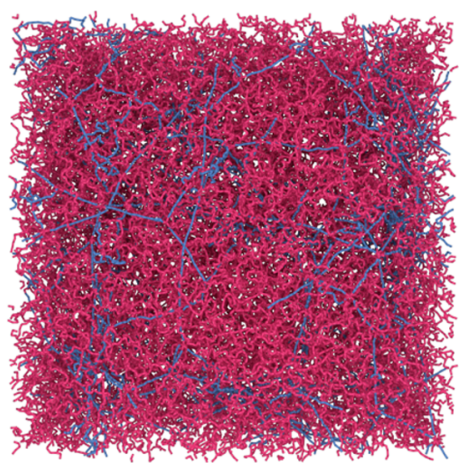

Figure 1. In silico double network generation. The sacrificial network is swollen from (a) $\lambda_{0}=1.00$ to (b) $\lambda=\lambda_{0}$. In this example, $\lambda_{0}=2.00$. (c) Monomers for the matrix are added at random positions in the swollen sacrificial network such that the number density of the entire system equals $\rho$. (d) The matrix is formed within the sacrificial network with the same cross-linking procedure used for the sacrificial network but with a lower cross-linker concentration.

main microscopic cause for the enhanced mechanical properties in the fracture regime. This microscopic picture for accumulation of damage is confirmed by experiments ${ }^{2,13,15,16}$ and simulations. ${ }^{17}$ However, despite state-of-the-art experimental techniques enabling the visualization of stress, ${ }^{18}$ strain, ${ }^{19-21}$ and the accumulation of damage at the local level, ${ }^{2,4,22}$ a thorough understanding of how the microscopic processes affect the global material response is still lacking.

Several constitutive models have been put forward that provide a connection between the evolution of damage and the global stress response of a $\mathrm{DN}^{23-27}$ These models can be fitted to experimental data and are also used in the interpretation of the output of mechanophores, i.e., molecular probes that report on the rupture of bonds locally. ${ }^{18}$ Some of these models, ${ }^{25-27}$ referred to as statistical damage mechanics models, predict the global response from the evolution of chain-stretch with respect to an initial stretch distribution, assuming affine deformation and breaking of overstretched chains. As a result, the global mechanical response of a DN predicted by these models is the sum of the responses of two (or more) independent and affinely deforming networks. Effectively, these models assume that (statistically or on average) the intranetwork load distribution follows the global deformation and that internetwork load redistribution is negligible at the global level. However, in the case of DN mechanics, these assumptions deserve some scrutiny because, at first glance, they seem to be incompatible with the proposed DN toughening mechanism where internetwork load sharing plays an essential role. In this work, we investigate to what extent the microscopic process of redistribution of load, both within and between networks, affects the global mechanical response.

To this end, we perform coarse-grained simulations of polymer networks, where load redistribution is intrinsically captured. To generate the DNs, we replicate a swelling procedure in silico, which is commonly used to make both hydrogel and elastomer DNs in the experiment. ${ }^{2,3,10}$ By deforming the networks, we obtain information on both the global stress response and the local stress, the local strain and the accumulation of damage. We show that the in silico networks behave in accordance with their experimental counterparts. Subsequently, we compare these simulation results with the affine predictions for the global stress and local damage response. From this comparison, we find that in our simulations the microscopic mechanism for damage accumulation differs significantly from the affine approximation, with the affine prediction overestimating the fraction of broken chains by almost an order of magnitude. Furthermore, we show that the accumulation of damage occurs in two steps, one controlled by load sharing within the sacrificial network and one by load sharing between the two networks. Finally, we show that in our simulations load sharing causes an enhanced global mechanical response, in contrast to the affine prediction. We conclude with a discussion of the implications of our findings for the microscopic picture of fracture in DNs and polymer networks in general.

\section{RESULTS AND DISCUSSION}

In Silico Preparation of DNs. We prepared our networks following the procedure of refs 28-30. In particular, the first network, or sacrificial network, is generated from 10000 

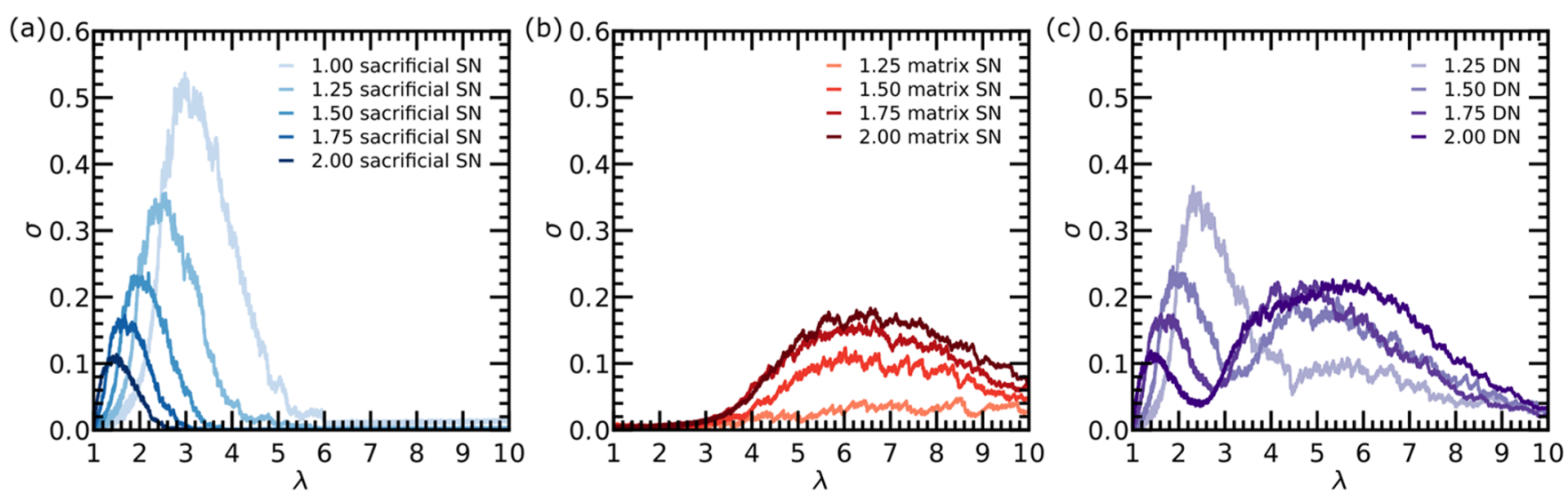

Figure 2. Mechanical response of SNs and DNs for a range of swelling ratios (see the legends). We plot the engineering stress $\sigma$ versus the global stretch $\lambda$ for (a) sacrificial SNs $\left(c_{1}=5 \%\right)$, (b) matrix SNs $\left(c_{2}=1 \%\right)$, and (c) DNs $\left(c_{1}=5 \%, c_{2}=1 \%\right)$. Note that the matrix SNs are generated by removing the sacrificial chains from the DNs.

particles of diameter $\sigma^{\prime}$ with a number density of $\rho=0.15$ (Figure 1a). The majority of these particles are bifunctional and can only form linear chains. A fraction $c_{1}=5 \%$ of the particles are tetrafunctional and can cross-link polymer chains. After network generation, we swell the sacrificial network isotropically up to a swelling ratio $\lambda_{0}=L_{\mathrm{box}} / L_{0, \mathrm{box}}$ (Figure $1 \mathrm{~b}$ ) and add particles for the second network or matrix (Figure 1c). The matrix is formed with a cross-linker fraction $c_{2}=1 \%$ (Figure 1d). Because $c_{2}<c_{1}$, the sacrificial polymer chains are shorter $\left(\langle L\rangle=10.9 \sigma^{\prime}\right)$ than the matrix chains $\left(\langle L\rangle=47.3 \sigma^{\prime}\right)$ in line with the empirical design rules for creating tough DNs. The distribution in chain lengths is exponential, as expected for a random polymerization process (see the Supporting Information for details). A detailed description of the procedure can be found in the Methods section. We have chosen the parameters based on a trade-off between experimental reality and feasibility of the simulations (see the Supporting Information for details).

Note that our protocol for creating DNs differs from other simulation works in several ways. Typically, polymer networks are formed by cross-linking preformed chains of given length, ${ }^{17,31-33}$ rather than using a random polymerizationlike procedure as we do. Furthermore, we prepare DNs via sequential polymerizations that conceptually resemble the experimental protocols, ${ }^{2,3}$ instead of the simultaneous assembly of both networks as previously done. ${ }^{17,31,32}$ There are other examples of in silico DNs generated by swelling. However, differently from our procedure, either internetwork cross-linking is allowed ${ }^{34}$ or only the bonds of cross-linkers are allowed to break. ${ }^{33}$

Mechanical Response of In Silico DNs. To obtain the mechanical response of our networks, we perform a uniaxial extension at a constant strain-rate and a constant volume, i.e., we impose a Poisson ratio $\nu=0.5$. We do this for both the DNs and the stand-alone networks. To facilitate comparison with the experimental work, we plot the engineering stress $\sigma$, which is calculated by dividing the deviatoric (true) stress by the global stretch $\lambda$ (see the Methods section for details). All results are reported in reduced (Lennard-Jones) units.

Following one of the curves in Figure $2 \mathrm{a}$ (e.g., $\lambda_{0}=1.00$ ), we can identify four mechanical regimes. After a short linear response at low strain (the linear elastic regime), the network becomes strain stiffening, as is expected for entropic springs, around $\lambda=1.50$ (the nonlinear elastic regime). Subsequently, strain-softening starts from $\lambda=2.25$, induced by breaking of chains, until the maximum strength $\sigma_{\max }$ of the sacrificial $\mathrm{SN}$ is reached at $\lambda=3.35$ (the strain-softening regime). After this point, the stress drops rapidly, indicating that significant damage is done to the network, cracks start to propagate, and the capability to carry load is lost (the crack propagation regime). Swelling the networks (without adding the matrix) increases the linear modulus (see the Supporting Information) and decreases the onset of strain stiffening, the stretch at the maximum strength and the maximum strength. All of these effects can be explained by the fact that network swelling leads to prestretching of the polymer chains, so that polymers are tensed already before applying uniaxial deformation, and less additional stress is needed to induce strain stiffening and rupture. This has been observed also in experiments. ${ }^{4,10}$ Note that at the largest swelling ratio $\lambda_{0}=2.00$, a few sacrificial chains break already during the swelling procedure $(\sim 0.5 \%$ of all chains).

For the stand-alone matrix networks (matrix SN), obtained by removing the sacrificial network, we find the same mechanical regimes as for the sacrificial SN (Figure 2b). However, as on average the matrix chains are longer than the sacrificial chains, the onset of strain stiffening and the maximum stress is found at higher strains. Because the matrix networks are formed after the swelling procedure (and thus carry no significant prestretch), we do not find a significant shift in the onset of strain stiffening or the strain at maximum strength with $\lambda_{0}$. We do find a dependency of $\sigma_{\max }$ on $\lambda_{0}$, which is caused by the increase in the matrix monomer density $\rho_{2}$ with the swelling ratio: $\rho_{2}=\rho-\rho_{1}=\rho\left(1-1 / \lambda_{0}^{3}\right)$. In other words, the polymer chain density in the matrix increases with $\lambda_{0}$, providing more chains to resist elongation.

The mechanical response of the DNs (Figure 2c) is clearly influenced by both the sacrificial network, which dominates at low strain, and the matrix network, which dominates at high strain. The loop in stress at intermediate strains marks the transition between these two regimes. A similar transition is observed for some hydrogels and elastomers in experiments in the form of a plateau with a constant stress after a certain "yield" point. ${ }^{4,10}$ Such an extended fracture response is atypical for hydrogels and elastomers, which normally fracture in an abrupt manner after reaching their maximum strength, i.e., brittle fracture. In analogy to the fracture response of various metals, this extended fracture response is referred to as ductile. 


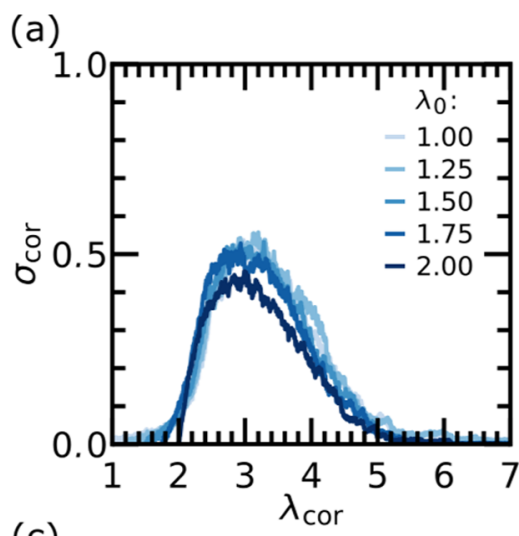

(b)
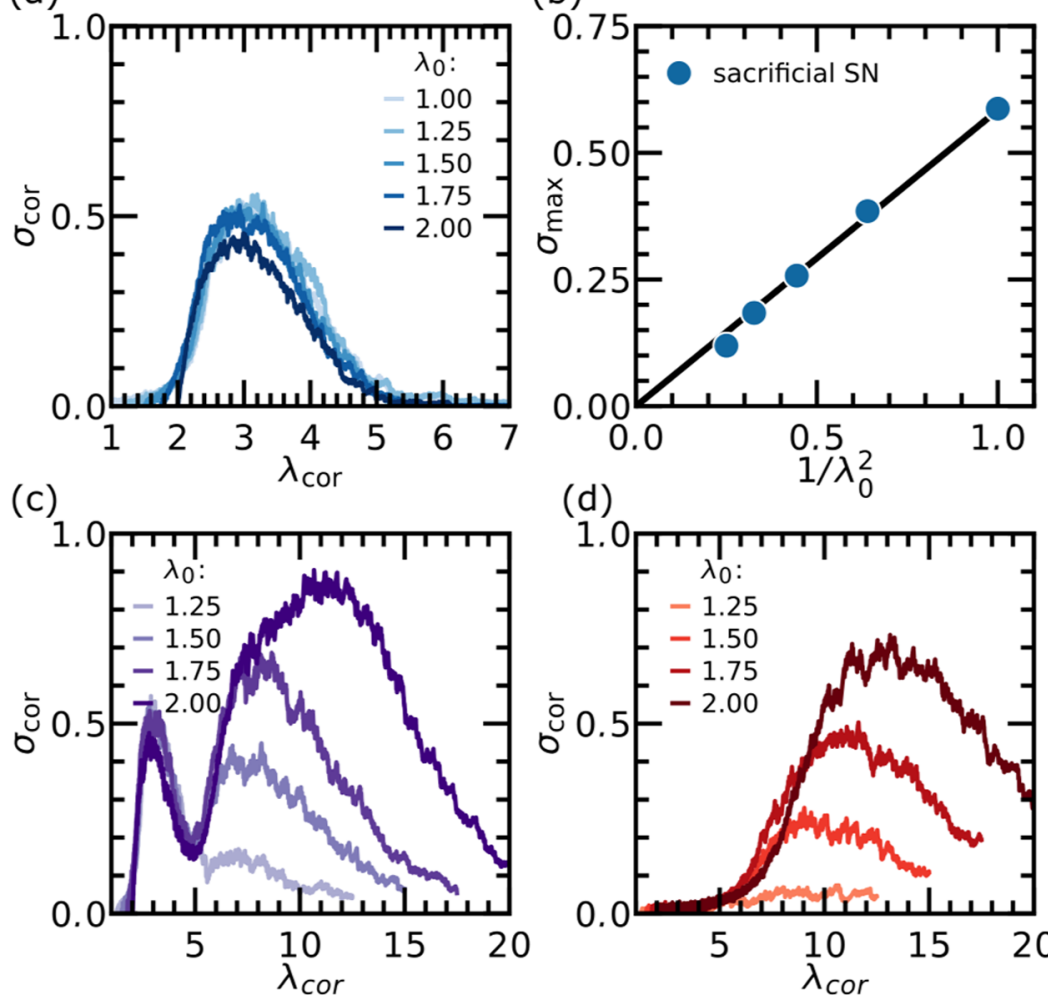

(d)

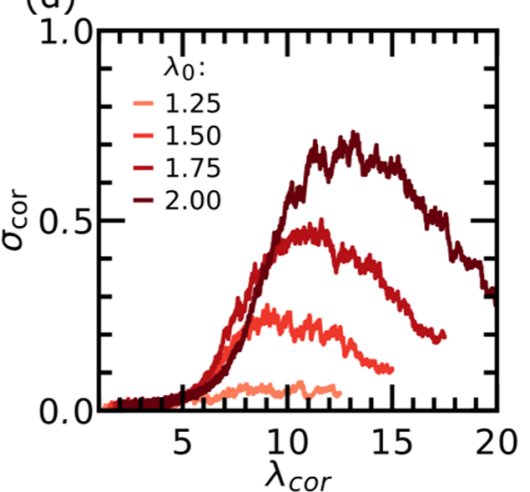

Figure 3. Initial stress response is controlled by the sacrificial network. (a) Rescaled stress-strain curves of sacrificial SNs $\left(c_{1}=5 \%\right.$ ), with $\sigma_{\text {cor }}=\sigma \lambda_{0}^{2}$ and $\lambda_{\text {cor }}=\lambda \lambda_{0}$. (b) Maximum stress of sacrificial SN versus $1 / \lambda_{0}^{2}$, a proxy for a real strand density. (c) Rescaled stress - strain curves of DNs $\left(c_{1}=5 \%\right.$, $\left.c_{2}=1 \%\right)$. (d) Rescaled stress-strain curves of the matrix SNs $\left(c_{2}=1 \%\right)$.

In polymer double networks, this plateau is caused by the separation of the material into a soft and highly stretched region, in which many sacrificial bonds are broken, and a stiff and weakly stretched region, in which the sacrificial network is still intact, also referred to as necking. A force balance between these two regions causes the stress to be constant. We do not observe a plateau in our simulations because our networks are too small to get a separation into a soft (weakly stretched) and a stiff (highly stretched) region. In our simulations, the maximum strength of the material $\sigma_{\max }$ is determined by the stress peak either before or after the loop, depending on $\lambda_{0}$. We think this is indicative of the transition from brittle to ductile fracture, which is observed in the experiment as a function of $\lambda_{0}{ }^{4}$ For small $\lambda_{0}$, the first peak is highest, so that in an experimental setting the material will fracture abruptly once the local strain in any part of the network exceeds the strain at $\sigma_{\text {max }}$. However, for higher $\lambda_{0}$, when the second peak becomes higher than the first, a coexistence between regions of different (local) $\lambda$ becomes possible at stress equal to the peak stress of the first network, resulting in a ductile fracture response. The brittle-to-ductile transition would then occur at the point where both peaks are of the same height.

We already mentioned that the initial mechanical response of our DNs is dominated by the sacrificial network. Experimental work on hydrogels and swollen elastomers shows similar results ${ }^{4,35}$ and reveals that the experimental data can be rescaled onto a single master curve based on the areal strand density of the sacrificial network. ${ }^{4,10}$ Figure 3 reveals that also our simulation data can be collapsed on a master curve for both sacrificial SNs and DNs, confirming that at least up to the first peak in stress the response is dominated by the sacrificial network. The rescaling corrects for the increase in prestretch $\left(\lambda_{\text {cor }}=\lambda \lambda_{0}\right)$ and the reduction in areal strand density in the sacrificial network $\left(\sigma_{\text {cor }}=\sigma \lambda_{0}^{2}\right)$. The onset of strain stiffening in our rescaled curves occurs around $\lambda_{\text {cor }} \approx$ 1.5 , and the peak stress falls around $\lambda_{\text {cor }} \approx 3.0$. The latter value is close to the maximum extension limit of our chains under ideal conditions $\left(\lambda_{\text {limit }}=\sqrt{ } N=3.37\right.$ with $\left.\langle N\rangle=11.35\right)$.

The collapse for both DNs and sacrificial SNs shows that the maximum stress at the first peak is determined by the strength of the sacrificial network, resulting in the linear scaling between $\sigma_{\max }$ and $\lambda_{0}^{-2}$ shown in Figure 3b. In the experiment, a similar scaling was found for the yield stress in ductile DNs, ${ }^{4}$ implying that in the experiment the yield stress is determined by the strength of the sacrificial network. However, unlike our simulations, this linear scaling is only found at high $\lambda_{0}$. At low $\lambda_{0}$, the experimentally measured yield (or breaking) stress increases with $\lambda_{0}{ }^{4}$ Our explanation for this experimental observation is that the fracture strength of polymer networks is not only determined by the areal strand density but also by the presence of defects. Because stress concentrates around defects, their presence can drastically reduce the global stress at which macroscopic cracks are formed and global failure is induced. In DNs, the effect of these defects in the sacrificial network is mitigated because the expansion of the defects into macroscopic cracks is hampered by the matrix chains. An increase in $\lambda_{0}$ increases the volume fraction of matrix chains and thus increases the screening effect. As a result, the yield (or breaking) stress will increase with $\lambda_{0}$ as long as global failure is induced by defects in the sacrificial network. Only at high $\lambda_{0}$, when most defects are screened by the matrix chains, the areal strand density will dominate the fracture response, leading to 
(a)

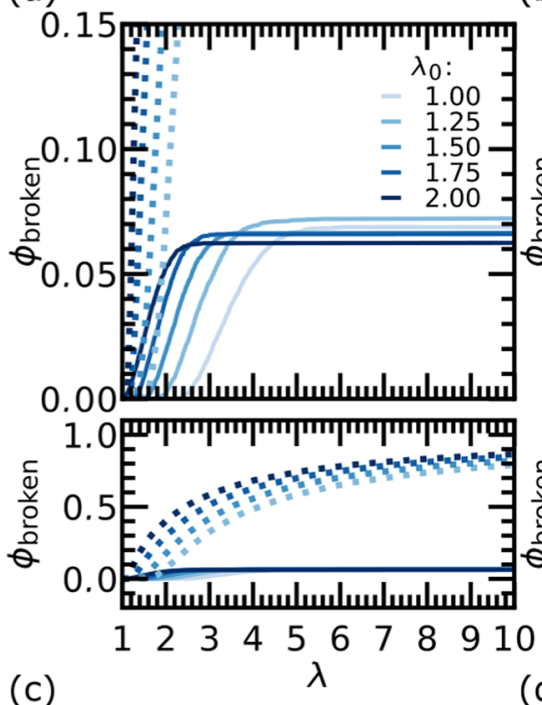

(c)

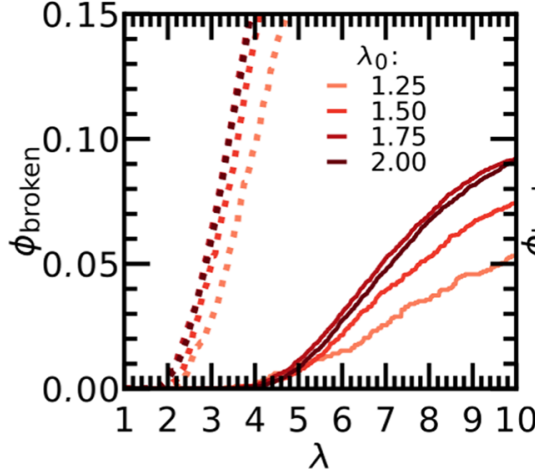

(b)

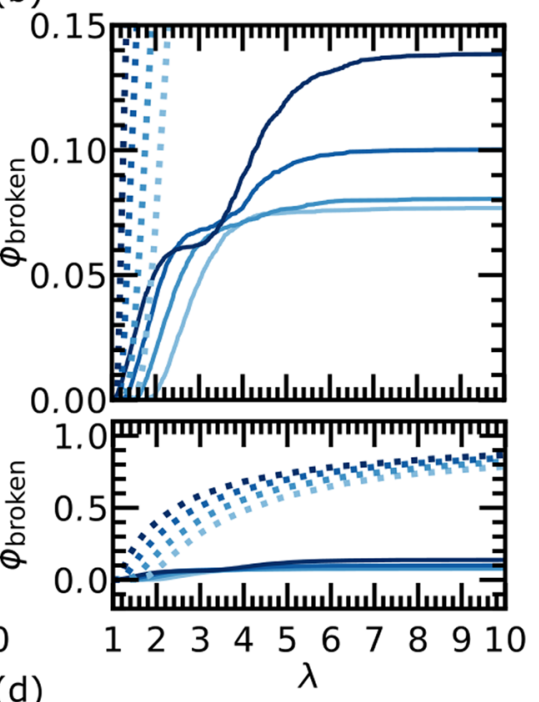

(d)

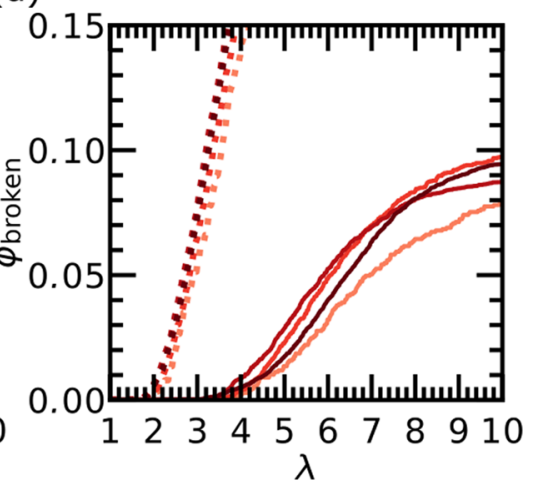

Figure 4. Fraction of broken chains $\phi_{\text {broken }}$ as a function of global stretch. The affine prediction for $\phi_{\text {broken }}$ is indicated with the dashed lines. (a) $\phi_{\text {broken }}$ for sacrificial chains in the sacrificial SN $\left(c_{1}=5 \%\right)$ for a range of $\lambda_{0}$ as indicated in the legend. To illustrate the large difference between the affine prediction and the simulation results, we plot the curves for the entire range of $\phi_{\text {broken }}$ in the lower panel and for a smaller range in the upper panel. (b) $\phi_{\text {broken }}$ for sacrificial chains in DNs $\left(c_{1}=5 \%, c_{2}=1 \%\right)$ for the same swelling ratios as panel (a). (c) $\phi_{\text {broken }}$ for matrix chains in matrix SNs $\left(c_{2}=1 \%\right)$ for a range of $\lambda_{0}$ as indicated in the legend. (d) $\phi_{\text {broken }}$ of matrix chains in DNs $\left(c_{1}=5 \%, c_{2}=1 \%\right)$ for the same swelling ratios as panel (c).

the expected decrease in the yield stress with increasing $\lambda_{0}$. In our simulations, we do not observe this sensitivity to defects because our networks are too small to contain defects that can dominate the fracture response.

On passing, we note that the postpeak response of the simulated networks is more ductile than for experimental ones. This is a finite-size effect, also observed in elastic spring networks. $^{36}$ In the remainder, we therefore focus on the prepeak behavior, which we have shown to be consistent with the experimental observations. Furthermore, we note that networks formed at a higher number density of $\rho=0.34$ behave in a similar way to networks formed at $\rho=0.15$ (see the Supporting Information for details).

Deviations from Affine Deformation. The goal of this work is to assess whether the process of inhomogeneous load redistribution affects the global response and the process of damage accumulation at the local level. To quantify this effect, we compare the results of our simulations, where load sharing is intrinsically captured, with an affine prediction for the response, which by definition does not take into account the interaction between the networks. The simulations more closely resemble the experimental reality, where the local load distribution is a result of internetwork rearrangements and excluded volume interactions between networks, while the affine prediction assumes that the local deformation exactly follows the globally applied deformation, implying that interactions within and between networks have a negligible effect on the global response. We make these affine predictions based on the evolution of the distribution in end-to-end distances under affine deformation, similar to the statistical damage mechanics models discussed earlier. For easy comparison between chains of different lengths, we introduce the dimensionless chain-stretch $r / L$ with $r$ being the end-toend distance and $L$ being the total chain length. We assume that the polymers break at an average chain-stretch of $r / L=$ 1.129 , which corresponds to the stretch where the activation barrier for bond rupture is equal to the thermal energy ${ }^{37}$ (see the Supporting Information for details). Note that in our simulations for sacrificial SNs, chains break around $r / L=1.08$. We attribute this lower value to the presence of topological constraints, which can lead to an underestimation of the chain tension based on the end-to-end distance. Since the contribution of each polymer to the affine stress-strain response is independent, crack nucleation or propagation cannot be captured in the affine prediction. Our comparison, therefore, focuses on the damage accumulation regime prior to $\sigma_{\max }$ 
(a)

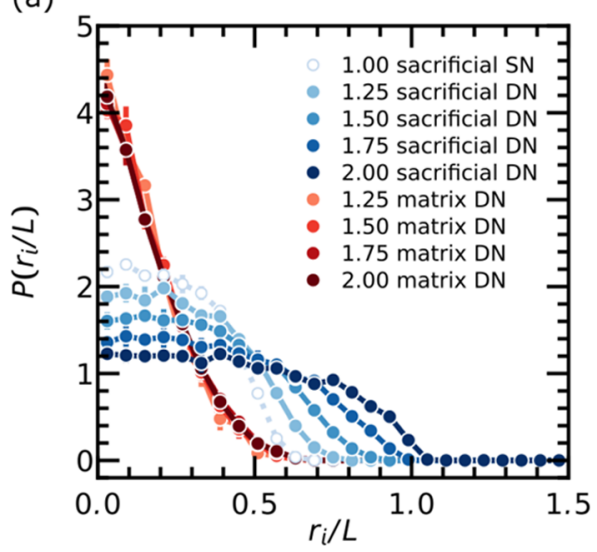

(b)

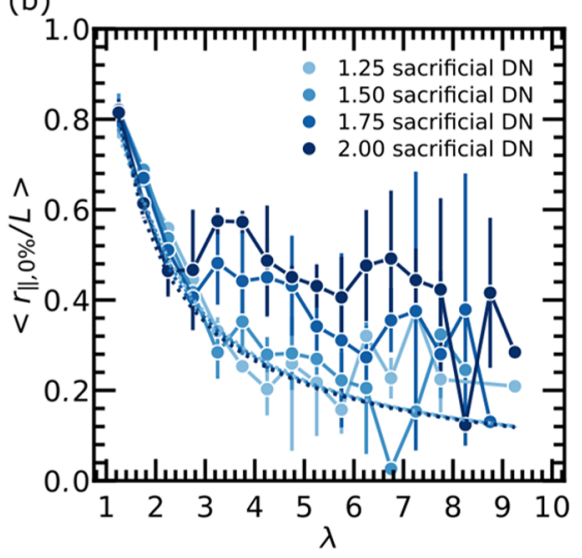

(c)

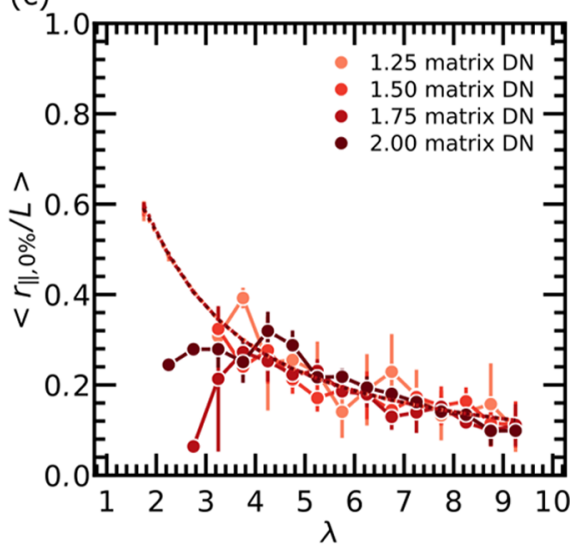

Figure 5. Role of initial chain-stretch, or prestretch, in chain scission. (a) Chain prestretch distribution at $0 \%$ strain for swollen sacrificial networks embedded in a matrix (blue), as well as for matrix networks formed inside swollen sacrificial networks (red). The swelling ratios $\lambda_{0}$ are indicated in the legend. In addition to the data for DNs (solid symbols), the prestretch distribution of the sacrificial network prior to swelling $\left(\lambda_{0}=1.00\right)$ is shown (open symbols). Here, the stretch $r_{i} / L$ is the end-to-end distance in one direction divided by the contour length of the polymer. The distributions in these plots are based on $r_{i} / L$ in all three dimensions. (b, c) Average initial chain-stretch along the direction of applied strain $\left\langle r_{\|, 0 \%} /\right.$ $L\rangle$ of broken chains as a function of the global strain $\lambda$ at chain rupture for (b) sacrificial DNs and (c) matrix DNs. The dashed lines represent the affine prediction. In all plots, the error bars indicate the standard deviation over four configurations.

Damage Accumulation. From the microscopic point of view, the main characteristic of the double network response is the enhanced fracture of sacrificial chains. Indeed, we observe a significant increase in the fraction of broken sacrificial chains $\phi_{\text {broken }}$ in the DN (Figure $4 \mathrm{~b}$ ) compared to that in the SN (Figure 4a). The enhancement increases with $\lambda_{0}$, reaching up to a $100 \%$ increase for $\lambda_{0}=2.00$. This observed enhancement is a clear indication that in our simulations on DNs the sacrificial network interacts with the matrix network.

Comparing the simulation data (solid lines) with the affine predictions (dashed lines), we find that the affine prediction overestimates $\phi_{\text {broken }}$ by almost an order of magnitude for both the sacrificial SN and sacrificial DN (see the bottom panels in Figure $4 a, b$ ). In the affine prediction, the behavior of all chains in a single network is considered to be independent, i.e., if a chain breaks, this has no effect on the stress carried by the neighboring chains. The significant overestimation of $\phi_{\text {broken }}$ by the affine models implies that in our simulation interactions at the network level play an important role in the failure response. The simplest way to introduce network structure into the affine model would be to consider global failure when percolation is lost. However, in such a simple model, the fraction of broken chains is still too high with respect to our simulations ( $\phi_{\text {broken }}$ $\approx 0.99) .{ }^{38}$ In fact, the fraction of broken chains observed in our simulations is closer to the fractions observed for the failure of athermal elastic networks, ${ }^{36,39}$ where the fracture response is controlled by network rigidity. ${ }^{36,40-42}$

Going back to our simulation data, we also find that the rate of chain failure (the slope of the curves) drops significantly at the start of the transition regime in the stress-strain curve for DNs (Figure 3c), implying that in a DN the fracture of sacrificial chains takes place in two steps. This is in sharp contrast to the affine prediction where the development of $\phi_{\text {broken }}$ is the same for both the sacrificial SN and the sacrificial DN due to the absence of interactions between the two networks.

Combining these insights, we hypothesize that the two-step fracture mechanism in our simulations is controlled by network interactions. The first step is controlled by interaction within the sacrificial network, while the second step is controlled by the topological constraints between the sacrificial network and the matrix. A drop in the fracture rate has been observed experimentally for elastomers, ${ }^{4}$ and also experiments on hydrogels identified more than one fracture regime..$^{13}$ Finally, we note that in our simulations the rupture of matrix chains does not take place in two steps; however, the fracture of matrix chains occurs earlier in DN networks than in the matrix SN (Figure 4c,d).

Which Chains Are Likely to Break? The next step in studying the microscopic fracture response is to ask whether we can predict which chains will break. A good first guess would be that shorter chains are likely to break at a lower global strain than longer ones because for an ideal chain, the average stretch at break scales as $\mathrm{Nb} /(\sqrt{ } \mathrm{Nb})=\sqrt{ } \mathrm{N}$. However, we do not find this trend for the sacrificial network in either the simulation results or the affine prediction (see the Supporting Information). This is because in a network the average end-to-end distance of a polymer is constrained by the connections with other chains in the network. This results in a distribution in the average chain-stretch, or prestretch, especially in disordered networks with a distribution in chain length and local connectivity. In Figure $5 \mathrm{a}$, we show the distribution in prestretch as the distribution in $r_{i} / L$, which is the end-to-end distance along one axis, divided by the contour length $L$ of the polymer.

Considering this distribution in average prestretch, we could hypothesize that instead of the chain length the chain-stretch at $0 \%$ strain determines when a chain will break, so that the sequence in which bonds break can be predicted from the initial chain-stretch distribution. This is also assumed in the statistical damage mechanics approach. ${ }^{25}$ In Figure $5 b, c$, we plot the average prestretch at $0 \%$ strain $\left\langle r_{\|, 0 \%} / L\right\rangle$ of broken chains as a function of the global stretch $\lambda$ at which the chains break including both the simulation results (solid lines) and the affine prediction (dashed lines). For the sacrificial network, we indeed find that at low strains, the initial chain-stretch does scale with the global strain at break, just as for the affine prediction. For SNs, this is true for almost all broken chains (see the Supporting Information). However, in DNs, this correlation becomes weaker with an increasing $\lambda_{0}$ and for high 
(a)
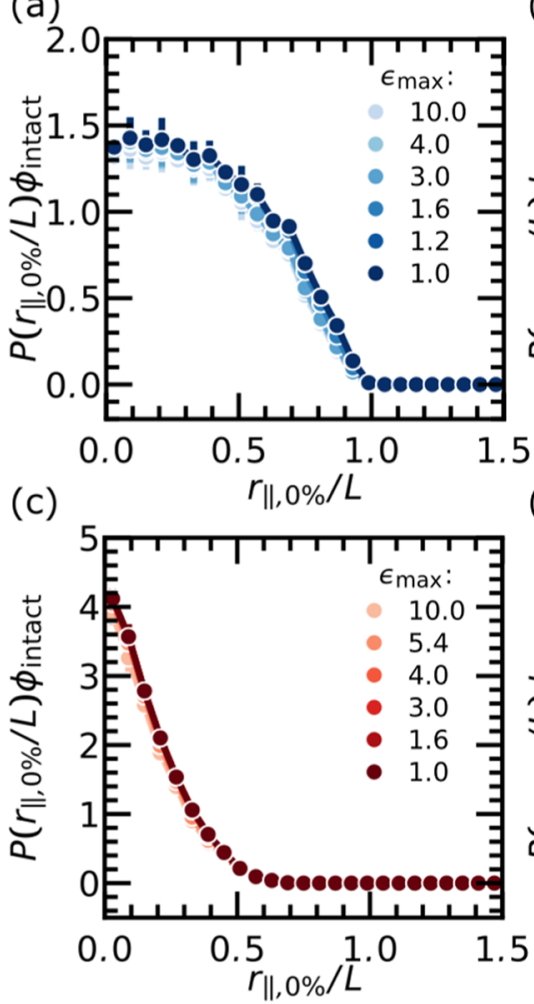

(b)

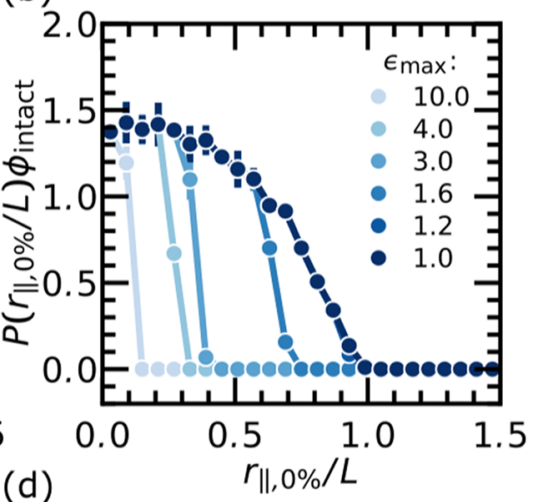

(d)

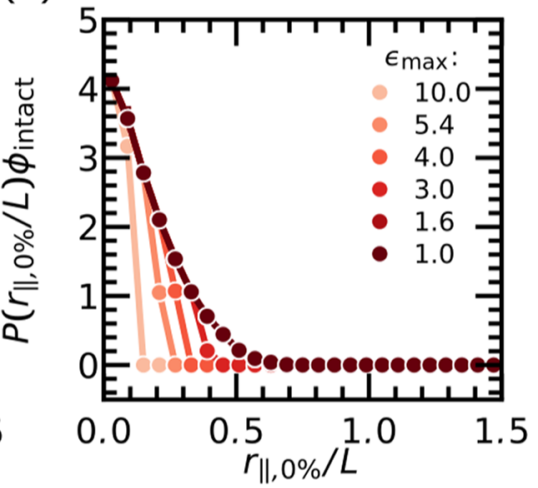

Figure 6. Predictability of chain rupture based on initial average chain-stretch. (a) Distribution in initial chain-stretch for all intact sacrificial chains in a DN at $\lambda_{0}=1.75$ at a maximum strain $\epsilon_{\max }$. The distributions are scaled by the fraction of intact chains with respect to the initial configuration. (b) Affine prediction for the sacrificial chains in the DN. (c) Distribution in initial chain-stretch for all intact matrix chains in a DN at a maximum strain $\epsilon_{\max }$ (d) Affine prediction for matrix chains in a DN.

$\lambda_{0}$ the correlation even seems to be lost after the first peak stress (this point also corresponds to the minimum in the chain rupture rate). This observation indicates that up to the peak stress the breaking of chains is largely defined by the configuration at $0 \%$ strain, i.e., network rearrangements do not affect the tension on the chains that break before the peak. However, after this peak stress (the start of the transition region in the $\mathrm{DN}$ ), the initial structure no longer controls which sacrificial chains break. As a consequence, the initial chain-stretch is not a predictor of failure anymore and the interactions with the matrix (i.e., topological constraints) dominate. This interpretation aligns with our hypothesis that network fracture takes place in two steps.

Note that the initial chain-stretch distribution of the sacrificial networks is determined by the structure of the network and the level of swelling $\lambda_{0}$. In our simulations, the evolution of chain-stretch with $\lambda_{0}$ is largely affine with respect to the distribution at $\lambda_{0}=1.00$ (see the Supporting Information). We also find that the initial chain-stretch distribution of both the sacrificial and matrix networks is the same in the $\mathrm{SN}$ and the $\mathrm{DN}$, indicating that prior to deformation, interactions between the networks are negligible. Note that for the matrix polymers we do find a correlation between chain length and breaking strain in both the simulation results and the affine prediction; this is because there is a wider distribution in chain lengths in the matrix networks (see the Supporting Information).

Which Chains Do Break? The observation that the initial chain-stretch is predictive for when a bond breaks over a large strain-range (Figure 5), similar to the affine prediction, is surprising considering the enormous overestimation of broken chains by the affine approximation (Figure 4). To investigate what is going on, we plot the distribution of the initial chainstretch for all of the intact chains at a particular strain (Figure 6). We find that although the initial chain-stretch is predictive for when a chain can break, this does not mean that all chains with that initial chain-stretch will break. Actually, only a few of those chains break, which is in sharp contrast with the affine prediction (Figure 6b). Our explanation is that in the first failure regime the deformation is largely affine in the undamaged network, but once a polymer breaks, significant stress relaxation becomes possible via rearrangements at the local level, alleviating the tension on polymers that surround the broken chain. In other words, we expect that stress heterogeneity within the network grows once damage starts to accumulate. Similar behavior is observed for the rupture of matrix chains, as shown in Figure 6c,d.

Evolution of the Prestretch Distribution. The data on the accumulation of damage suggest that in our simulations inhomogeneous redistribution of stress is taking place both within single networks during the first failure process and between networks during the second failure process. As the stress distribution within a polymer network is strongly related to the distribution in chain-stretch, we expect that any inhomogeneous mode of stress redistribution should be reflected in the evolution of the chain-stretch distribution as a function of strain. Furthermore, by comparing the evolution of chain-stretch in our simulations, where intra- and internetwork interactions are accounted for, with the affine prediction for the evolution of chain-stretch, where intra- and internetwork interactions are neglected, we can identify if and when network level processes affect the mechanical response of 
polymer networks. In particular, we look at the distribution in end-to-end distances parallel to the axis of deformation $r_{\|} / L$ (Figure 7), as the chain-stretch along the direction of applied deformation is primarily responsible for the global mechanical response.

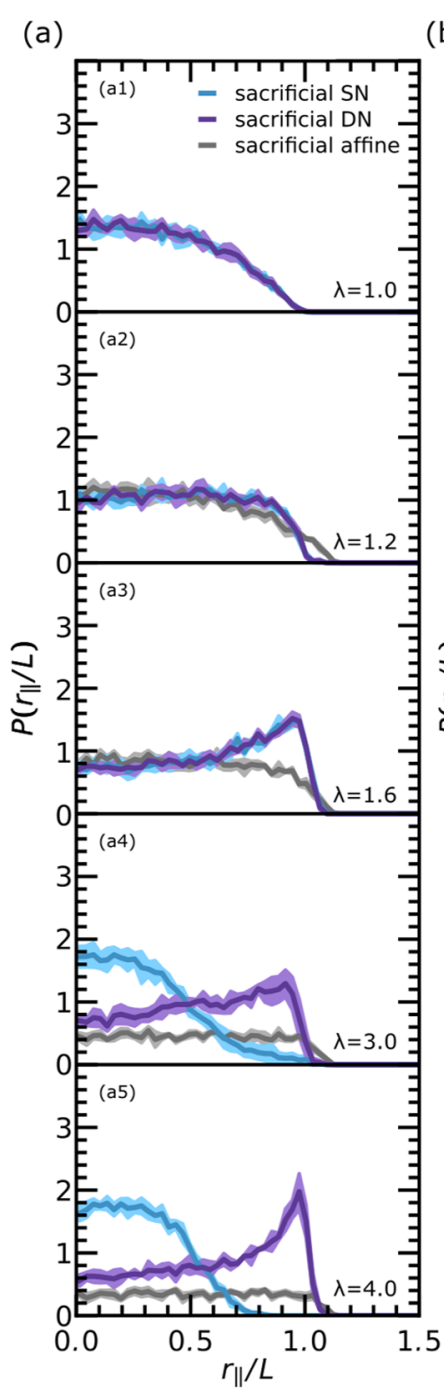

(b)

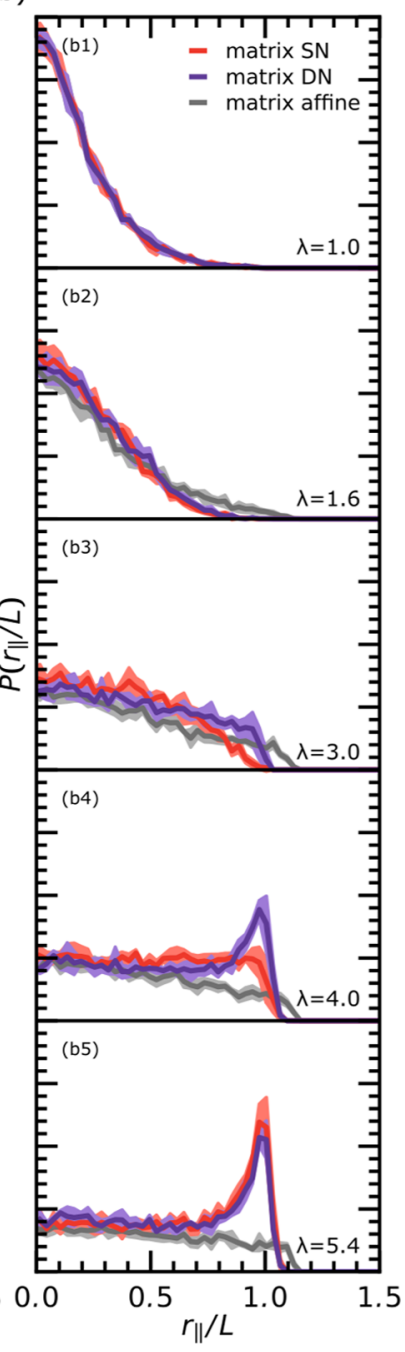

Figure 7. Evidence of network-network interaction based on stretch distribution. Comparison of chain-stretch in a network (SN and DN) at different strains as indicated in the plot $\left(\lambda_{0}=1.75\right)$. (a) Sacrificial network as a stand-alone network ( $\mathrm{SN}$, blue) and as part of a DN (purple). (b) Matrix network as a stand-alone network (SN, red) and as part of a DN (purple). For both panels, the affine prediction for the chain-stretch distribution is indicated in gray. The shading indicates the standard error computed over the four configurations.

When the strain is increased from $\lambda=1.0$ to 1.2 for a network at $\lambda_{0}=1.75$, we see that the distribution for both the sacrificial SN and the sacrificial DN flattens, in a similar way as expected for affine deformation (Figure 7a2). Around the first peak stress at $\lambda=1.6$ (Figure $7 \mathrm{a} 3$ ), we see an accumulation of chains that are stretched up to their contour length $\left(r_{\|} / L=\right.$ 1.0) in both the sacrificial SN and sacrificial DN. This behavior is very different from the response expected based on affine deformation, where no peak is visible, and implies that in our simulations additional load imposed on strongly stretched chains does not always lead to chain rupture (as in the affine prediction) but can also lead to redistribution of load to less stretched polymer chains. This mode of inhomogeneous stress redistribution might be caused by local stress relaxation after chain rupture, as discussed in the previous section. As the same trend is observed in sacrificial SN and sacrificial DN chains this is a clear sign of inhomogeneous redistribution of load within the network.

At higher strains $(\lambda=3.0$ and 4.0 ; Figure $7 \mathrm{a} 4, \mathrm{a} 5)$, the behavior of sacrificial chains in the $\mathrm{DN}$ diverges from the behavior in SNs. While the sacrificial chains in the SN relax to a stretch below the initial chain-stretch and remain there at higher strains as a result of macroscopic network fracture, a large part of the sacrificial chains in the DN remain close to their entropic stretching limit $\left(r_{\|} / L=1.0\right)$. At $\lambda=4.0$ (Figure $7 \mathrm{a} 5)$, the number of chains at maximum extension increases again in the DN. The divergence between the $\mathrm{SN}$ and $\mathrm{DN}$ chain-stretch demonstrates the effect of adding a matrix on the microscopic stress distribution within the network, revealing that due to internetwork interactions, sacrificial chains are still under significant tension beyond the first peak stress. Only far beyond the second peak in stress $(\lambda=10.0)$, the sacrificial chains start to relax, as strongly stretched chains rupture (not shown) due to the macroscopic fracture of the DN.

In the matrix network (Figure $7 \mathrm{~b}$ ), we observe that initially the $\mathrm{SN}$ and DN behave in the same way, but between a stretch of $\lambda=3$ and 4 , we see that there are more stretched chains in the $\mathrm{DN}$ compared to those in the $\mathrm{SN}$, indicating that during the transition regime, an interaction between the two networks arises. These data also match the shift in the stress response we have seen earlier for the matrix DN compared to those in the matrix SN (Figure 2). Overall, it becomes clear that in our simulations we find inhomogeneous stress redistribution at the network level at low strains and inhomogeneous stress redistribution between networks at high strains. These different processes for stress management explain the two distinct failure regimes identified for the sacrificial chains in Figure 4.

Impact on Global Response. So far, we have shown that the microscopic response of the networks is dominated by processes of inhomogeneous stress redistribution both within single networks and between networks. With increasing strain, these microscopic processes deviate further from the affine picture. The question that remains is: do these processes only matter at the local level or do they also affect the global stress response? To answer this question, we make a prediction for the stress response under affine deformation based on the prestretch distributions shown previously, assuming that the resistance to deformation of a single chain can be described as an extensible freely jointed chain (eFJC; see the Methods section for details). Although our short chains are not expected to behave exactly as ideal chains, a cross-check of this method with the simulation result reveals that this assumption still serves our purpose (see the Supporting Information for details).

Comparing the affine prediction with the simulation results, we find that in the linear regime, the affine prediction agrees quite well with the simulations at low swelling ratios (see the Supporting Information). For the response at larger strains, we look at the networks for $\lambda_{0}=1.75$ in Figure $8 \mathrm{a}, 8 \mathrm{~b}$. We immediately see that in the affine prediction (dotted lines) strain stiffening sets in earlier than in our simulations (solid lines) both for the SNs (Figure 8a) and for the DN (Figure $8 \mathrm{~b})$. This suggests that in the nonlinear elastic regime network rearrangements reduce the tension on individual polymers. 
(a)

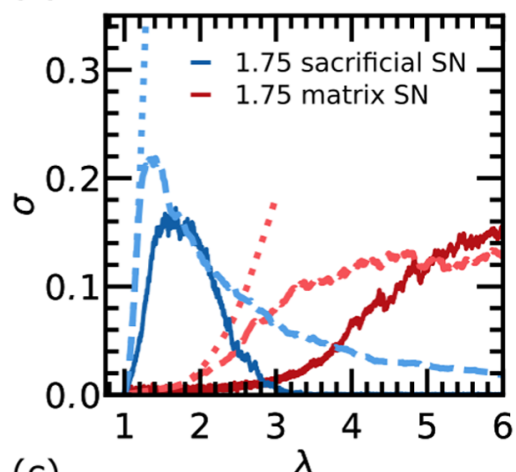

(c)

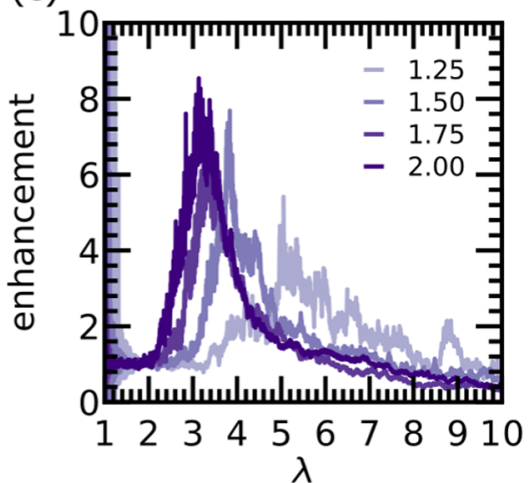

(b)

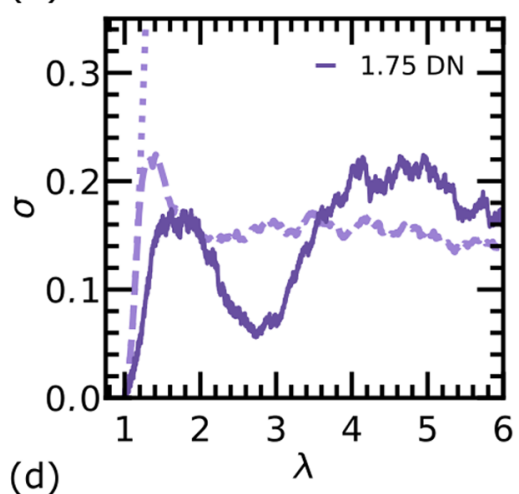

(d)

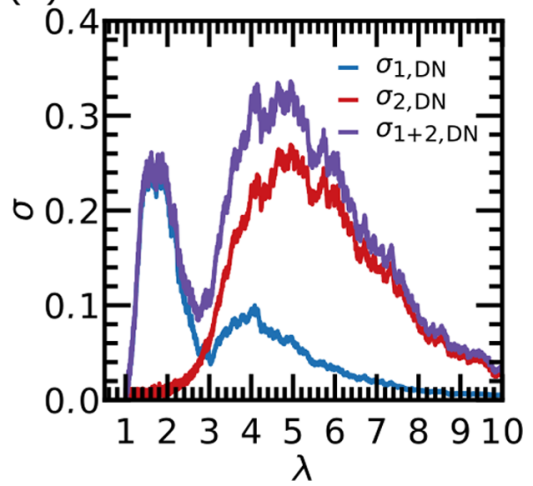

Figure 8. Effect of load sharing on the global stress response. (a, b) Comparison of the stress response from simulation with the affine prediction $\left(\lambda_{0}=1.75, c_{1}=5 \%, c_{2}=1 \%\right)$. The stress response upon affine deformation is determined from the average cross-linker positions at $0 \%$ strain, assuming that the stress carried by the polymer in between the cross-linkers can be described as a freely jointed chain with extensible quartic bonds (eFJC; see the Methods section for details). (a) SN response of the sacrificial network (blue) and the matrix network (red) from simulation (solid line), and the affine prediction for the mechanical response (dotted line) and the affine prediction considering polymers with $r / L>1.129$ to be broken (dashed line). (b) DN response. (c) Enhancement in measured stress in the DN $\sigma_{1+2, \mathrm{DN}} /\left(\sigma_{1, \mathrm{SN}}+\sigma_{2, \mathrm{SN}}\right)$ with $\sigma_{1+2, \mathrm{DN}}$ being the measured stress in the $\mathrm{DN}, \sigma_{1, \mathrm{SN}}$ being the measured stress in the sacrificial $\mathrm{SN}$, and $\sigma_{2, \mathrm{SN}}$ being the measured stress in the matrix SN. Swelling ratios are indicated in the legend. (d) Stress response of the sacrificial network $\sigma_{1, \mathrm{DN}}$ (blue) and the matrix network $\sigma_{2, \mathrm{DN}}\left(\right.$ red) embedded in the DN at $\lambda_{0}=$ 1.75. For reference, we also plot the total stress response of the $\mathrm{DN} \sigma_{1+2, \mathrm{DN}}$ (purple).

Even though the impact on the global stress is significant, calculation of the nonaffine displacement of the cross-links shows that in most cases these rearrangements are relatively small in the nonlinear elastic regime (see the Supporting Information). Because of the nonlinear stress response of single polymer chains, a small rearrangement can still have a significant impact on stress, providing a possible explanation for the strong effect observed here.

We find that the stress of the affine prediction quickly overshoots the simulation response if bond breaking is not considered for both SNs and DNs (dotted lines). If instead, we assume that chains with a stretch larger than 1.129 break, we find an affine $\mathrm{SN}$ response (dashed lines) in Figure $8 \mathrm{a}$ that is qualitatively similar to the simulation results (solid lines). However, in the affine case, the onset of strain-softening occurs earlier, the maximum stress is higher, the strain at maximum stress is lower, and the stress drops after the maximum stress is smoother. These differences imply that inhomogeneous redistribution of load within a network upon breaking of chains has a significant impact on the global stress response. The picture is the same if we compare the affine prediction for the behavior of the DN (dashed line) with the simulation result (solid line) in Figure 8b. We note that the loop observed in the affine case is less pronounced than in the simulations. We attribute this to the broad distribution in chain length, which causes a relatively smooth decay of the affine stress in the sacrificial network (Figure 8a) and the relatively small difference in cross-linker density between the sacrificial network and the matrix.

We have found several indications from the microscopic response in our simulations that interactions arise between the two networks if the strain is high enough (Figures 4, 5, and 7). To check if these network-network interactions affect the global stress response, we compare the stress of the DN with the sum of the stresses for the individual $\mathrm{SNs}$ via the enhancement factor $\sigma_{1+2, \mathrm{DN}} /\left(\sigma_{1, \mathrm{SN}}+\sigma_{2, \mathrm{SN}}\right)$ (Figure $8 \mathrm{c}$ ). We observe that significant enhancement starts after a certain stretch and that the onset of enhancement decreases as a function of $\lambda_{0}$. The onset of enhancement seems to coincide with the peak strain of the sacrificial SN (Figure 2). After the onset of enhancement, the enhancement factor increases up to a factor of 8.0 at the peak. Also, the location of this peak decreases as a function of $\lambda_{0}$. The enhancement peaks just after the end of the transition region. If we plot the stress of the sacrificial network and the matrix network in the DN $\left(\sigma_{1, \mathrm{DN}}\right.$ and $\sigma_{2, \mathrm{DN}}$ in Figure $8 \mathrm{~d}$ ) together with the total stress $\sigma_{1+2, \mathrm{DN}}$, we see that the enhancement in stress contains contributions of both the sacrificial network and the matrix, further confirming that the enhancement is caused by the interaction between the two networks. The second peak in stress in the sacrificial DN response suggests that even after the yield stress, sacrificial chains resist deformation. Indeed, we find that around this 
second peak in stress, a considerable fraction of the sacrificial chains is stretched up to their contour length (Figure 7a5).

\section{CONCLUSIONS AND OUTLOOK}

Our simulations confirm that in a $\mathrm{DN}$, both the local and global responses are governed by sharing of load at the network level. Similar to experiments, ${ }^{4}$ the mechanical response and accumulation of damage at low strains are governed by the sacrificial network even in the strain-softening regime. The behavior at the microscopic level reveals that upon deformation and damage accumulation stress is redistributed inhomogeneously within the network via small and local nonaffine rearrangements.

After the yield stress, the mechanical response is controlled by both the sacrificial network and the matrix network. The enhancement in broken chains (Figure 4), the change in failure mechanism (Figure 5), and the altered stretch distributions (Figure 7) reveal that both networks interact with each other through their topological constraints, leading to large nonaffine rearrangements at the network level. These internetwork interactions cause an enhancement in the fraction of broken sacrificial chains, in line with the sacrificial bond principle. At the global level, these interactions cause a significant enhancement in the stress response of DNs compared to those of the SNs (Figure 8).

The comparison of our simulation data with affine predictions suggests that for any polymer network ( $\mathrm{SN}$ or $\mathrm{DN}$ ) the inhomogeneous redistribution of load through the network can be an important mechanism in the nonlinear elastic and fracture response. In the nonlinear elastic regime, nonaffine rearrangements appear to be small. Therefore, we expect that in this regime the rearrangements are mainly driven by the nonlinear stress response of entropic springs in combination with the disordered structure of the network. However, in the fracture regime, we find strong deviations from the affine prediction. In particular, we observe that a significant fraction of the chains is stretched beyond their contour length (Figure 7). Experiments on single polymer chains also reveal that the extension of polymer chains up to this limit is possible. ${ }^{37}$ These data suggest that in the fracture regime enthalpic stretching could play an important role in the behavior of networks prior to the propagation of a macroscopic crack.

Our simulations provide predictions for the effect of load sharing on the microscopic fracture response. Several of these predictions can be verified in the experiment. For example, the low fraction of broken chains (Figure 4) could be investigated by quantification of the fraction of broken chains prior to crack propagation by incorporating chain scission reporters in the network such as dioxetane ${ }^{2}$ or anthracene. ${ }^{43}$ Our simulations also suggest that the distribution in chain-stretch provides information on the (inhomogeneous) redistribution of load within a network and between networks (Figures 5 and 7). Although tracking the evolution of the chain-stretch distribution would be a challenging endeavor, experiments on Förster resonance energy-transfer (FRET)-based force-sensors show that this might be possible experimentally. ${ }^{44}$

\section{METHODS}

In Silico Synthesis of Double Network. The networks are formed by the self-assembly of binary mixtures of bifunctional and tetrafunctional patchy particles, as done in refs $28-30$. To build the first network, we simulate the binary mixture at a number density $\rho_{\text {init }}$
$=0.17$. We stop the simulation when most $(>99.9 \%)$ of the bonds have formed, after which we remove the few clusters that are not attached to the largest one. No more than $3 \%$ of the particles are removed at this stage. We take the resulting system, locate all of the chains, defined as clusters of bifunctional particles connecting the cross-linkers, and add five monomers to each to make the system more swellable (resulting in a number density $\rho_{\text {init,add }}=0.33$ ).

To swell the network in LAMMPS, ${ }^{45}$ we convert the network of patchy particles to a network of harmonic bonds and equilibrate the network in the NVT ensemble for $10 \tau$. Subsequently, we convert the harmonic bonds to quartic bonds and equilibrate the network in the NPT ensemble for $100 \tau$ such that the network settles at an equilibrium box size $L_{\mathrm{box}, 0}$. The resulting network is the sacrificial SN at $\lambda_{0}=1.00$ and number density $\rho=0.15$. This network is swollen isotropically (NVT ensemble) in steps of $\sim 0.1 \%$ strain such that the new box size is $L_{\mathrm{box}}=L_{\mathrm{box}, 0} \lambda_{0}$, providing the sacrificial SNs at higher swelling ratios.

To form the corresponding DNs, we add matrix monomers and subsequently perform the same self-assembly procedure described above, with the difference that this time the bifunctional and tetrafunctional particles are embedded in the existing polymer network. After the assembly of the binary mixture completes, we once again remove the few disconnected clusters but this time we do not add any additional monomers to the chains. The resulting DNs are NVT-equilibrated in LAMMPS first using harmonic bonds (for $10 \tau$ ) and then using quartic bonds (for $10 \tau$ ). Finally, the matrix SNs are obtained by removing the sacrificial network from the DNs.

For all LAMMPS simulations, the time step for integration $\mathrm{d} t=$ $0.001 \tau$. For simulations performed in the NVT ensemble, the temperature is controlled via a Nosé-Hoover thermostat and kept fixed at $T=1.0$ (in reduced units) with a damping time $t_{\text {damp }}=0.1 \tau$ (100 time steps). In addition, for simulations performed in the NPT ensemble, the pressure is fixed at $P=1.0$ (in reduced units) and the corresponding $t_{\text {damp }}=1.0 \tau$ (1000 time steps).

The interaction between the particles is described by the WeeksChandler-Andersen (WCA) potential, a truncated version of the Lennard-Jones potential

$$
U(R)=4 €\left[\left(\frac{\sigma^{\prime}}{R}\right)^{12}-\left(\frac{\sigma^{\prime}}{R}\right)^{6}\right], R<R_{\mathrm{c}}
$$

with $R$ the interparticle distance, $\sigma^{\prime}=1.0$ is the particle diameter, $\epsilon=$ 1.0 is the depth of the potential well, and $R_{c}=2^{1 / 6}$ is the cutoff distance, unless the particles are connected by a bond; in that case, the particle-particle interaction is described by a quartic potential ( $K$ $\left.=2351, B_{1}=-0.7425, B_{2}=0.0, R_{c}=1.5, U_{0}=92.74467\right)$

$$
\begin{aligned}
U(R)= & K\left(R-R_{\mathrm{c}}\right)^{2}\left(R-R_{\mathrm{c}}-B_{1}\right)\left(R-R_{\mathrm{c}}-B 2\right) \\
& +U_{0}+4 \epsilon\left[\left(\frac{\sigma^{\prime}}{R}\right)^{12}-\left(\frac{\sigma^{\prime}}{R}\right)^{6}\right]+\epsilon
\end{aligned}
$$

These parameters have been used previously to study polymer rupture. ${ }^{46}$ The quartic bonds break irreversibly if their extension exceeds $R_{c}=1.5$. However, the maximum force is already reached around an extension of $1.133 \sigma^{\prime}$ and the bonds are expected to break even earlier, around an extension of $1.08 \sigma^{\prime}$, due to thermal fluctuations (see the Supporting Information for details). In some equilibration steps, harmonic bonds are used instead of a quartic bond $\left(K=1000, R_{0}=0.96\right)$

$$
U(R)=K\left(R-R_{0}\right)^{2}
$$

Extension Protocol. The stress response is obtained by performing a continuous uniaxial extension at a strain-rate $\dot{\epsilon}=1 \times$ $10^{-4}$ while keeping the volume of the simulation box constant (lateral dimensions are reduced during extension). A similar procedure has been followed in the literature. ${ }^{33,34}$ Decreasing the strain-rate by a factor of 10 does not significantly alter the mechanical response. The stress response $\sigma$ is determined from the virial stress excluding kinetic contributions, which are nevertheless negligible. First, we calculate the 
deviatoric (true) stress as $\sigma_{\mathrm{T}}=\sigma_{i i}-\sigma_{\text {hydr }}$. Subsequently, we convert this to the engineering stress $\sigma=\sigma_{\mathrm{T}} / \lambda$. For every configuration, the deformation protocol is performed in the $x, y$, and $z$ directions and the output is averaged. Data presented in the manuscript are averages over four configurations. If error bars are used, they indicate the standard deviations in the values between these four configurations.

Analysis of Chains. Polymer chains are defined as the set of particles in between cross-linkers, the latter having connectivity different from 2. Some of the $N_{\text {chains }}$ in a network are trivial dangling ends, i.e., one of the ends of the chain has functionality of 1 , and are indicated as $N_{\text {dang. }}$. We also identified (first-order) loops when both chain ends share the same cross-linker, and we indicated these as $N_{\text {loops }}$. Both loops and dangling ends are expected to not contribute to the mechanical response; therefore, in a first approximation, we can define the active chains as $N_{\text {act }}=N_{\text {chains }}-N_{\text {dang }}-N_{\text {loops. }}$. We define the chain length, $L$, as $b \times\left(N_{\text {beads }}-1\right)$, where $b=0.96$ is the rest length of the quartic bond and $N_{\text {beads }}$ is the number of particles in a polymer chain including the cross-linkers. We define the end-to-end distance $r$ as the Euclidean distance between cross-linkers. To calculate $r$ prior to deformation, we use the average cross-linker positions from a simulation run of $10000 \tau$, where the cross-linker locations are saved every 50 $\tau$. For the calculation of $r$ during deformation, the cross-linker positions are based on snapshots, which are saved every $\Delta \lambda=0.01$. In both cases, coordinates are unwrapped to correct for periodic boundary crossings and corrected with respect to their combined center of mass. We consider a polymer chain to be broken if one of the bonds inside the chain breaks. Breaking of bonds is reported via a custom extension of the LAMMPS code.

Affine Predictions. Affine predictions for $r_{i} / L$ distributions and stress are made based on the time-averaged positions of chain ends of active chains, i.e., cross-linkers. Based on this configuration, we can determine the average location of chain ends after affine deformation. From these positions, we calculate the end-to-end distances of all of the polymer chains. Chains are considered broken if their chainstretch exceeds the maximum stretch of a quartic bond $(1.08 / b=$ 1.129). Broken chains are not included in the distributions.

To predict the stress response, we assume that the stress response of the single polymers can be described as an extensible freely jointed chain (eFJC), ${ }^{47}$ which covers both entropic and enthalpic contributions. In this way, we can obtain the stress contribution of every polymer based on the location of the chain ends. Combining the contributions of all active polymers, we get our prediction of the virial stress tensor and thus the global response based on affine deformation.

To find the stress contribution of every polymer, we rewrite the chain-stretch as $r / L=\left(\left(r_{L}\right) /(L)\right) \lambda_{b}$, where $r_{L} / L$ is the entropic chainstretch and $\lambda_{\mathrm{b}}=R_{\mathrm{b}} / b$ is the enthalpic stretch of a quartic bond. $\lambda_{\mathrm{b}}$ is found by numerically solving the force balance $\frac{\mathrm{d} U_{\text {quartic }}\left(\lambda_{\mathrm{b}}\right)}{\mathrm{d} \lambda_{\mathrm{b}}} \lambda_{\mathrm{b}}=k_{\mathrm{B}} T \frac{r}{\lambda_{\mathrm{b}} L} \mathcal{L}^{-1}\left(\frac{r}{\lambda_{\mathrm{b}} L}\right)$ according to ref 47 , where $\mathcal{L}^{-1}$ is the inverse Langevin equation (we use the approximation by Puso ${ }^{48}$ ). Based on this value, we can calculate the force from $F=\frac{k_{\mathrm{B}} T}{b \lambda_{\mathrm{b}}} \mathcal{L}^{-1}\left(\frac{r}{\lambda_{\mathrm{b}} L}\right)$ . Note that $k_{\mathrm{B}} T=1.0$ in reduced units.

\section{ASSOCIATED CONTENT}

\section{(s) Supporting Information}

The Supporting Information is available free of charge at https://pubs.acs.org/doi/10.1021/acs.macromol.1c01275.

Explanation of parameterization, additional network characterization, mechanical response for networks formed at $\rho=0.34$, and validation of the eFJC estimation for the stress carried by a coarse-grained polymer (PDF)

\section{AUTHOR INFORMATION}

\section{Corresponding Author}

Jasper van der Gucht - Physical Chemistry and Soft Matter, Wageningen University and Research, 6708 WE Wageningen, The Netherlands; 10 orcid.org/0000-0001-5525-8322; Email: jasper.vandergucht@wur.nl

\section{Authors}

Justin Tauber - Physical Chemistry and Soft Matter, Wageningen University and Research, 6708 WE Wageningen, The Netherlands; (1) orcid.org/0000-0002-1573-1987

Lorenzo Rovigatti - Dipartimento di Fisica, SapienzaUniversità di Roma, 00185 Roma, Italy; 이이.org/00000001-5017-2829

Simone Dussi - Physical Chemistry and Soft Matter, Wageningen University and Research, 6708 WE Wageningen, The Netherlands; John A. Paulson School of Engineering and Applied Sciences, Harvard University, Cambridge, Massachusetts 02138, United States

Complete contact information is available at:

https://pubs.acs.org/10.1021/acs.macromol.1c01275

\section{Notes}

The authors declare no competing financial interest.

\section{ACKNOWLEDGMENTS}

This work is part of the SOFTBREAK project funded by the European Research Council (ERC Consolidator Grant 682782).

\section{REFERENCES}

(1) Gong, J. P.; Katsuyama, Y.; Kurokawa, T.; Osada, Y. Doublenetwork hydrogels with extremely high mechanical strength. Adv. Mater. 2003, 15, 1155-1158.

(2) Ducrot, E.; Chen, Y.; Bulters, M.; Sijbesma, R. P.; Creton, C. Toughening elastomers with sacrificial bonds and watching them break. Science 2014, 344, 186-189.

(3) Nakajima, T.; Sato, H.; Zhao, Y.; Kawahara, S.; Kurokawa, T.; Sugahara, K.; Gong, J. P. A universal molecular stent method to toughen any hydrogels based on double network concept. Adv. Funct. Mater. 2012, 22, 4426-4432.

(4) Millereau, P.; Ducrot, E.; Clough, J. M.; Wiseman, M. E.; Brown, H. R.; Sijbesma, R. P.; Creton, C. Mechanics of elastomeric molecular composites. Proc. Natl. Acad. Sci. U.S.A. 2018, 115, 9110-9115.

(5) Tanaka, Y. A local damage model for anomalous high toughness of double-network gels. Europhys. Lett. 2007, 78, No. 56005.

(6) Brown, H. R. A model of the fracture of double network gels. Macromolecules 2007, 40, 3815-3818.

(7) Xin, H.; Saricilar, S. Z.; Brown, H. R.; Whitten, P. G.; Spinks, G. M. Effect of first network topology on the toughness of double network hydrogels. Macromolecules 2013, 46, 6613-6620.

(8) Ahmed, S.; Nakajima, T.; Kurokawa, T.; Anamul Haque, M.; Gong, J. P. Brittle-ductile transition of double network hydrogels: Mechanical balance of two networks as the key factor. Polymer 2014, 55, 914-923.

(9) Gong, J. P. Why are double network hydrogels so tough? Soft Matter 2010, 6, 2583-2590.

(10) Matsuda, T.; Nakajima, T.; Fukuda, Y.; Hong, W.; Sakai, T.; Kurokawa, T.; Chung, U. I.; Gong, J. P. Yielding Criteria of Double Network Hydrogels. Macromolecules 2016, 49, 1865-1872.

(11) King, D. R.; Okumura, T.; Takahashi, R.; Kurokawa, T.; Gong, J. P. Macroscale Double Networks: Design Criteria for Optimizing Strength and Toughness. ACS Appl. Mater. Interfaces 2019, 11, $35343-35353$. 
(12) Nakajima, T. Generalization of the sacrificial bond principle for gel and elastomer toughening. Polym. J. 2017, 49, 477-485.

(13) Nakajima, T.; Kurokawa, T.; Ahmed, S.; Wu, W. L.; Gong, J. P. Characterization of internal fracture process of double network hydrogels under uniaxial elongation. Soft Matter 2013, 9, 1955-1966.

(14) Tauber, J.; Dussi, S.; Van Der Gucht, J. Microscopic insights into the failure of elastic double networks. Phys. Rev. Mater. 2020, 4, No. 063603.

(15) Webber, R. E.; Creton, C.; Brown, H. R.; Gong, J. P. Large strain hysterisis and Mullins effect of tough double network hydrogels. Macromolecules 2007, 40, 2919-2927.

(16) Mai, T. T.; Matsuda, T.; Nakajima, T.; Gong, J. P.; Urayama, K. Distinctive Characteristics of Internal Fracture in Tough Double Network Hydrogels Revealed by Various Modes of Stretching. Macromolecules 2018, 51, 5245-5257.

(17) Higuchi, Y.; Saito, K.; Sakai, T.; Gong, J. P.; Kubo, M. Fracture Process of Double-Network Gels by Coarse-Grained Molecular Dynamics Simulation. Macromolecules 2018, 51, 3075-3087.

(18) Chen, Y.; Joshua Yeh, C.; Qi, Y.; Long, R.; Creton, C. From force-responsive molecules to quantifying and mapping stresses in soft materials. Sci. Adv. 2020, 6, No. eaaz5093.

(19) Fukao, K.; Nakajima, T.; Nonoyama, T.; Kurokawa, T.; Kawai, T.; Gong, J. P. Effect of Relative Strength of Two Networks on the Internal Fracture Process of Double Network Hydrogels As Revealed by in Situ Small-Angle X-ray Scattering. Macromolecules 2020, 53, $1154-1163$.

(20) Ducrot, E.; Montes, H.; Creton, C. Structure of Tough Multiple Network Elastomers by Small Angle Neutron Scattering. Macromolecules 2015, 48, 7945-7952.

(21) Van Der Kooij, H. M.; Dussi, S.; Van De Kerkhof, G. T.; Frijns, R. A.; Van Der Gucht, J.; Sprakel, J. Laser Speckle Strain Imaging reveals the origin of delayed fracture in a soft solid. Sci. $A d v$. 2018, 4, No. eaar1926.

(22) Matsuda, T.; Kawakami, R.; Nakajima, T.; Gong, J. P. Crack tip field of a double-network gel: Visualization of covalent bond scission through mechanoradical polymerization. Macromolecules 2020, 53, $8787-8795$.

(23) Zhao, X. A theory for large deformation and damage of interpenetrating polymer networks. J. Mech. Phys. Solids 2012, 60, 319-332.

(24) Liu, Y.; Zhang, H.; Zheng, Y. A Micromechanically Based Constitutive Model for the Inelastic and Swelling Behaviors in Double Network Hydrogels. J. Appl. Mech. 2016, 83, No. 021008.

(25) Vernerey, F. J.; Brighenti, R.; Long, R.; Shen, T. Statistical Damage Mechanics of Polymer Networks. Macromolecules 2018, 51, 6609-6622.

(26) Lavoie, S. R.; Millereau, P.; Creton, C.; Long, R.; Tang, T. A continuum model for progressive damage in tough multinetwork elastomers. J. Mech. Phys. Solids 2019, 125, 523-549.

(27) Morovati, V.; Saadat, M. A.; Dargazany, R. Necking of doublenetwork gels: Constitutive modeling with microstructural insight. Phys. Rev. E 2020, 102, No. 062501.

(28) Gnan, N.; Rovigatti, L.; Bergman, M.; Zaccarelli, E. In Silico Synthesis of Microgel Particles. Macromolecules 2017, 50, 8777-8786.

(29) Rovigatti, L.; Gnan, N.; Zaccarelli, E. Internal structure and swelling behaviour of in silico microgel particles. J. Phys.: Condens. Matter 2018, 30, No. 044001.

(30) Sorichetti, V.; Ninarello, A.; Ruiz-Franco, J. M.; Hugouvieux, V.; Kob, W.; Zaccarelli, E.; Rovigatti, L. Effect of Chain Polydispersity on the Elasticity of Disordered Polymer Networks. Macromolecules 2021, 54, 3769-3779.

(31) Jang, S. S.; Goddard, W. A.; Kalani, Y. S. Mechanical and transport properties of the poly(ethylene oxide)-poly (acrylic acid) double network hydrogel from molecular dynamic simulations. J. Phys. Chem. B 2007, 111, 1729-1737.

(32) Li, H.; Wu, H.; Li, B.; Gao, Y.; Zhao, X.; Zhang, L. Molecular dynamics simulation of fracture mechanism in the double interpenetrated cross-linked polymer. Polymer 2020, 199, No. 122571.
(33) Yin, Y.; Bertin, N.; Wang, Y.; Bao, Z.; Cai, W. Topological origin of strain induced damage of multi-network elastomers by bond breaking. Extreme Mech. Lett. 2020, 40, No. 100883.

(34) Wang, W.; Zhang, Z.; Davris, T.; Liu, J.; Gao, Y.; Zhang, L.; Lyulin, A. V. Simulational insights into the mechanical response of prestretched double network filled elastomers. Soft Matter 2017, 13, $8597-8608$

(35) Nakajima, T.; Kurokawa, T.; Furukawa, H.; Gong, J. P. Effect of the constituent networks of double-network gels on their mechanical properties and energy dissipation process. Soft Matter 2020, 16, $8618-8627$

(36) Dussi, S.; Tauber, J.; Van Der Gucht, J. Athermal Fracture of Elastic Networks: How Rigidity Challenges the Unavoidable SizeInduced Brittleness. Phys. Rev. Lett. 2020, 124, No. 018002.

(37) Wang, S.; Panyukov, S.; Rubinstein, M.; Craig, S. L. Quantitative Adjustment to the Molecular Energy Parameter in the Lake-Thomas Theory of Polymer Fracture Energy. Macromolecules 2019, 52, 2772-2777.

(38) Li, Z.; Liu, Z. The elongation-criterion for fracture toughness of hydrogels based on percolation model. J. Appl. Phys. 2020, 124, No. 215101.

(39) Deogekar, S.; Islam, M. R.; Picu, R. C. Parameters controlling the strength of stochastic fibrous materials. Int. J. Solids Struct. 2019, 168, 194-202.

(40) Driscoll, M. M.; Chen, B. G. G.; Beuman, T. H.; Ulrich, S.; Nagel, S. R.; Vitelli, V. The role of rigidity in controlling material failure. Proc. Natl. Acad. Sci. U.S.A. 2016, 113, 10813-10817.

(41) Berthier, E.; Kollmer, J. E.; Henkes, S. E.; Liu, K.; Schwarz, J. M.; Daniels, K. E. Rigidity percolation control of the brittle-ductile transition in disordered networks. Phys. Rev. Mater. 2019, 3 , No. 075602.

(42) Tauber, J.; Kok, A. R.; Van Der Gucht, J.; Dussi, S. The role of temperature in the rigidity-controlled fracture of elastic networks. Soft Matter 2020, 16, 9975-9985.

(43) Slootman, J.; Waltz, V.; Yeh, C. J.; Baumann, C.; Göstl, R.; Comtet, J.; Creton, C. Quantifying Rate-and Temperature-Dependent Molecular Damage in Elastomer Fracture. Phys. Rev. X 2020, 10, No. 041045.

(44) van de Laar, T.; Schuurman, H.; van der Scheer, P.; Maarten van Doorn, J.; van der Gucht, J.; Sprakel, J. Light from Within: Sensing Weak Strains and FemtoNewton Forces in Single Molecules. Chem 2018, 4, 269-284.

(45) Plimpton, S. Fast parallel algorithms for short-range molecular dynamics. J. Comput. Phys. 1995, 117, 1-19.

(46) Ge, T.; Pierce, F.; Perahia, D.; Grest, G. S.; Robbins, M. O. Molecular dynamics simulations of polymer welding: Strength from interfacial entanglements. Phys. Rev. Lett. 2013, 110, No. 098301.

(47) Mao, Y.; Talamini, B.; Anand, L. Rupture of polymers by chain scission. Extreme Mech. Lett. 2017, 13, 17-24.

(48) Jedynak, R. Approximation of the inverse Langevin function revisited. Rheol. Acta 2015, 54, 29-39. 